\title{
Yersinia pseudotuberculosis infection in Kawasaki disease and its clinical characteristics
}

Tomoko Horinouchi ${ }^{1}$, Kandai Nozu ${ }^{2 *}$, Kiyoshi Hamahira', Yosuke Inaguma ${ }^{1}$, Jun Abe ${ }^{3}$, Hiroshi Nakajima ${ }^{4}$, Masaaki Kugo ${ }^{1}$ and Kazumoto lijima ${ }^{2}$

\begin{abstract}
Background: The etiology of Kawasaki disease (KD) is unknown. Reportedly, there is an association between KD and Yersinia pseudotuberculosis (YPT). Steroid therapy for KD patients with high risk of cardiac sequelae (CS) has been reported; however, the number of reports is limited.

Methods: We conducted a prospective study of 108 patients with newly diagnosed KD in one year to determine how many KD patients have positive anti-YPT antibody titers and/or positive anti-YPT-derived mitogen (YPM) antibody titers. In addition, we tried to identify clinical differences between KD patients in whom YPT infection was or not a contributing factor. We also compared clinical characteristics of patients treated with the protocol of the Randomized controlled trial to Assess Immunoglobulin plus Steroid Efficacy for Kawasaki disease (RAISE) study (RAISE group) and with the conventional Intravenous immunoglobulin (IVIG) protocol (conventional group).

Results: Eleven patients (10\%) were positive for anti-YPT and/or anti-YPM antibodies (positive group) and 97 (90\%) were negative (negative group). Cardiac sequelae (CS) occurred significantly more frequently in the positive than the negative group (two patients, $18 \%$ vs one patient, $1 \%, p=0.027$ ). Forty patients were in the RAISE group. Two of $40(5 \%)$ in the RAISE group and one of $68(1.47 \%)$ in the conventional group had CS $(p=0.55)$.

Conclusions: KD patients with YPT infection had CS significantly more frequently and treatment with RAISE protocol did not decrease the frequency of CS in our cohort, nor did YPT infection affect risk scores of no response to IVIG. However, our sample size was overly small to draw such conclusions. Further investigation in a larger cohort is necessary to confirm our findings. Additionally, further research is needed to determine whether early diagnosis of YPT can prevent KD from developing and reduce the incidence of CS.
\end{abstract}

\section{Background}

Kawasaki disease (KD), a febrile vasculitis of unknown origin, can cause coronary artery dilation and is increasing in incidence worldwide [1-3]. Identification of the cause and optimal treatment is urgently needed.

Yersinia pseudotuberculosis (YPT), an enteric pathogen, causes a variety of clinical symptoms such as fever, rash, desquamation, strawberry tongue, lymphadenopathy, and conjunctivitis that sometimes satisfy the clinical criteria for KD. Some research groups have reported an association between YPT and KD [4-7]. YPT is commonly

\footnotetext{
* Correspondence: nozu@med.kobe-u.ac.jp

${ }^{2}$ Department of Pediatrics, Kobe University Hospital, Kobe, Japan

Full list of author information is available at the end of the article
}

typed serologically according to antigenic differences in the lipopolysaccharide $\mathrm{O}$ antigen. There are 15 serogroups; serotypes O:1 and O:2 have subtypes a, b, and c, and serotypes O:4 and O:5 have subtypes a and b. Thus, there are 21 serotypes in all $[8,9]$. Serotypes O:1a, O:1b, O:2a, O:2b, O:2c, O:3, O:4b, O:5a, O:5b, O:6, O:10, and O:15 are known to be pathogenic for humans [10]. The pathogenicity of YPT is determined by a number of virulence factors, including a plasmid associated with Yersinia virulence, a high-pathogenicity island, and a Y. pseudotuberculosis-derived mitogen (YPM) [11-13]. Among these virulence factors, YPM is a superantigen that activates macrophages and $\mathrm{T}$ cells and causes symptoms such as high fever and erythema [14].

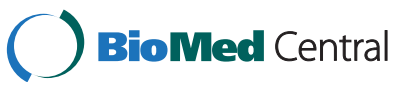

(c) 2015 Horinouchi et al. Open Access This article is distributed under the terms of the Creative Commons Attribution 4.0 International License (http://creativecommons.org/licenses/by/4.0/), which permits unrestricted use, distribution, and reproduction in any medium, provided you give appropriate credit to the original author(s) and the source, provide a link to the Creative Commons license, and indicate if changes were made. The Creative Commons Public Domain Dedication waiver (http://creativecommons.org/publicdomain/zero/1.0/) applies to the data made available in this article, unless otherwise stated. 
So far, few reports concerning the relationship between KD and YPT infection are available. The present study was designed to determine how often KD patients have associated YPT infection by assessing anti-YPT and antiYPM antibody titers and to identify clinical differences between KD patients with and without YPT infection.

Additionally, about $40 \%$ of KD patients in our cohort were treated with the RAISE (Randomized controlled trial to Assess Immunoglobulin plus Steroid Efficacy for Kawasaki disease) study protocol [15]. Clinical characteristics of patients treated with the RAISE protocol and the conventional IVIG (IntraVenous ImmunoGlobulin) protocol were compared to assess the effects of the RAISE study treatment strategy in patients with KD and the influence of YPT infection on treatment outcomes.

\section{Methods}

\section{Study patient definitions and design}

With approval of the Japanese Red Cross Society Himeji Hospital ethics committee, all KD patients hospitalized from April 2013 to March 2014 in the Japanese Red Cross Society Himeji Hospital were prospectively included in this study. Written informed consent for not only participating in this study but also to publish the patient data and information was obtained from all parents or guardians. Patients whose guardians refused consent or for whom blood samples for detection of anti-YPT and YPM antibodies were not available were excluded. Acute- and convalescent-phase sera were obtained from participants, who were then divided into two groups: a positive group (positive anti-YPT and/or positive anti-YPM antibody titers) and a negative group (both anti-YPT and anti-YPM antibody titers negative).

The following clinical data were recorded and compared between the two groups: sex, age, duration of fever in days, frequency of administration of high-dose IVIG, acute-phase coronary artery dilation, cardiac sequelae (CS), abdominal symptoms, white blood cell and absolute neutrophil counts, serum concentrations of albumin, total bilirubin, aspartate aminotransferase, sodium, C-reactive protein (CRP), procalcitonin, N-terminal probrain natriuretic peptide, and soluble interleukin-2 receptor, and urinary $\beta 2$-microglobulin/creatinine ratio.

\section{Measurement of anti-YPT antibody and anti-YPM antibody}

To identify and quantitate anti-YPT antibodies, lipopolysaccharide agglutination titers for serotypes $O: 1 a, 1 b, 2 a$, $2 b, 3,4 a, 4 b, 5 a, 5 b$, and 6 were measured by the Widal method. Sera positive for O:2 and/or O:4 were retested after absorption with Salmonella $\mathrm{O}$ groups four and nine, respectively, because the former have cross-Oantigen reaction against the latter $[16,17]$. The cut-off for positive anti-YPT antibodies was a single titer of 1:160 or higher [10].
Enzyme-linked immunosorbent assays were performed to measure anti-YPM antibodies, the optical density of non-antigen-coated wells being subtracted from that of antigen-coated wells. Anti-YPM antibody titers were determined using a calibration curve constructed from a positive control serum. Patients with a more than fourfold increase in serial antibody titers were considered positive for anti-YPM antibodies.

\section{Definition and treatments for KD}

Kawasaki disease was diagnosed in accordance with the Japanese Diagnostic Guidelines for Kawasaki Disease (5th edition) [17]. The initial treatment for KD consisted of IVIG $(2 \mathrm{~g} / \mathrm{kg})(n=108)$. A second dose of IVIG (2 g/ $\mathrm{kg}$ ) was given to patients whose fever had not resolved $\geq 48 \mathrm{~h}$ after receiving the first IVIG $(n=27)$. For patients whose fever had not resolved $\geq 48 \mathrm{~h}$ after receiving a second IVIG, additional treatments, including a third administration of IVIG $(2 \mathrm{~g} / \mathrm{kg})(n=12)$, infliximab $(5 \mathrm{mg} /$ $\mathrm{kg})(n=5)$, cyclosporin $(4 \mathrm{mg} / \mathrm{kg}$ per day, until CRP concentrations had reverted to normal) $(n=2)$, or methylprednisolone $(30 \mathrm{mg} / \mathrm{kg}$ per day, for 3 days) $(n=2)$ were considered. No patient underwent plasma exchange (Fig. 1). Almost all patients received aspirin $30 \mathrm{mg} / \mathrm{kg}$ per day until they were afebrile, followed by aspirin $3 \mathrm{mg} / \mathrm{kg}$ per day for 2 months after discharge from the hospital. Flurbiprofen $(4 \mathrm{mg} / \mathrm{kg} /$ day $)$ was substituted in patients with serum aspartate aminotransferase or alanine aminotransferase concentrations $\geq 200 \mathrm{U} / \mathrm{L}$.

Coronary artery dilation was defined as the internal diameter of the coronary artery being $>3 \mathrm{~mm}(>4 \mathrm{~mm}$ if the subject was over 5 years old) or the internal diameter of a segment being at least 1.5 times that of an adjacent segment according to echocardiography [18]. Acutephase coronary artery dilations were defined as those that normalized within 1 month and CS were defined as those remaining after 1 month [15].

\section{RAISE study group}

The treatment strategy was changed during the study period. At first, we started the KD treatment with the conventional IVIG protocol. However, we decided to change the treatment protocol, that is, we adopted the RAISE protocol, which reported clear evidence that steroids prevent CS in KD patients [15] and has already been adopted as a standard treatment in Japan which reflects the fact that this protocol has recently described in the guideline for treatment of KD in Japan [18, 19].

In response to the findings of the RAISE study, between December 2013 and March 2014, patients with risk scores $\geq 5$ points, which predicts failure to respond to initial treatment with IVIG, we adopted the RAISE protocol which reported clear evidence that intravenous prednisolone (PSL) $2 \mathrm{mg} / \mathrm{kg}$ per day (maximum dose of 


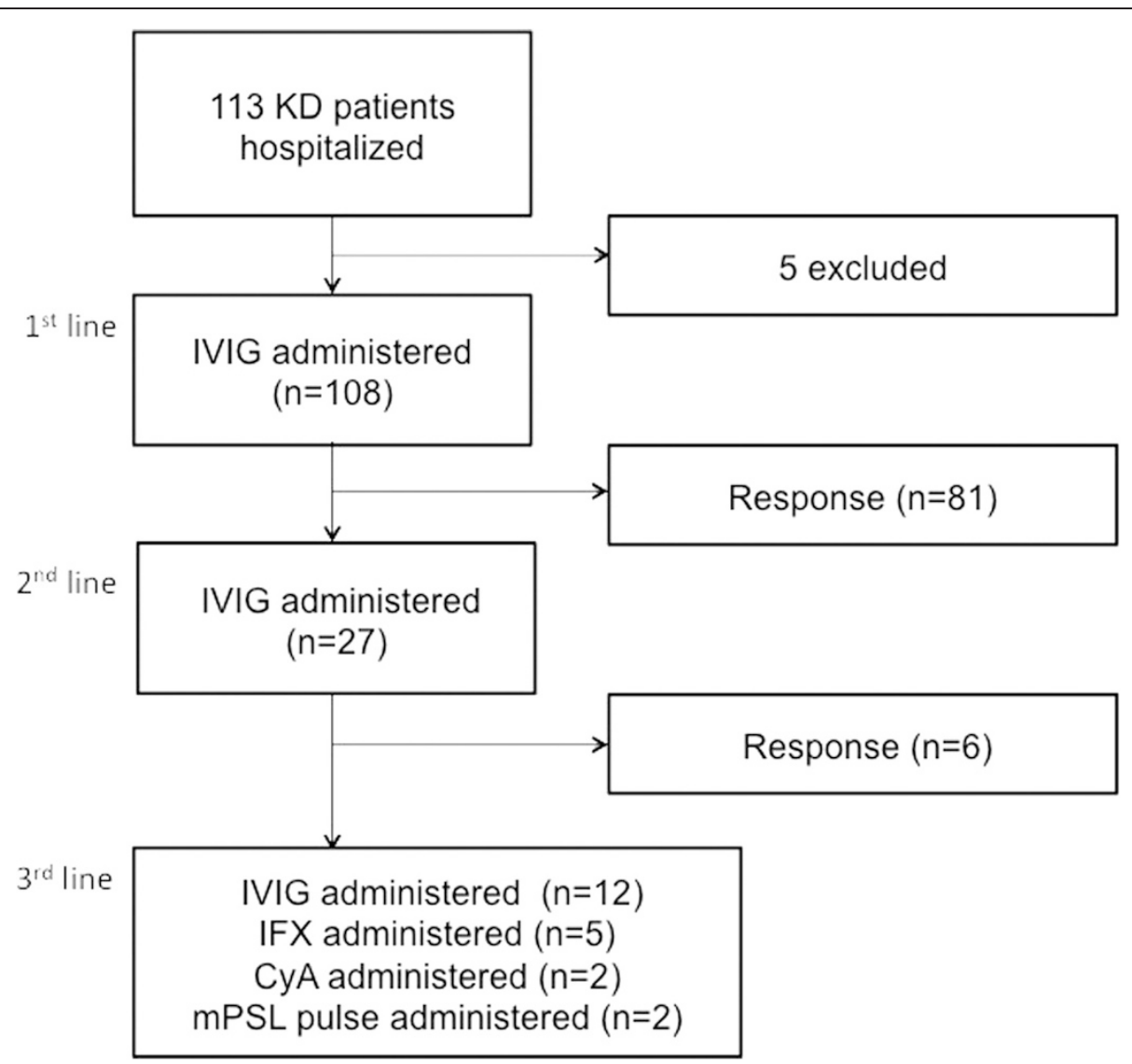

Fig. 1 Flow chart showing distribution of study subjects and medications used for KD. The numbers in parentheses denote the overall number of patients. KD: Kawasaki disease, IVIG: IntraVenous ImmunoGlobulin, IFX: Infliximab, CyA: cyclosporin, mPSL: methylprednisolone

$60 \mathrm{mg}$ per day) prevents CS in KD [15]. Once patients had become afebrile, PSL was administered orally. When serum CRP concentrations had decreased to within the normal range $(\leq 0.5 \mathrm{mg} / \mathrm{dL})$, the PSL dose was tapered over 15 days with the following dosage reductions at 5 day intervals: from $2 \mathrm{mg} / \mathrm{kg}$ per day to $1 \mathrm{mg} / \mathrm{kg}$ per day to $0.5 \mathrm{mg} / \mathrm{kg}$ per day. Scoring and cut-off values for risk scores were measured as described in the RAISE study, which is as follows: two points each for serum sodium concentration of $\leq 133 \mathrm{mmol} / \mathrm{L}, \leq 4$ days of illness at diagnosis, serum aspartate aminotransferase concentration of $\geq 100 \mathrm{U} / \mathrm{L}$, neutrophil count of $\geq 80 \%$; and 1 point each for platelet count $\leq 30 \times 10^{4} / \mu \mathrm{L}$, serum CRP concentration of $\geq 10 \mathrm{mg} / \mathrm{dL}$, and age $\leq 12$ months [15]. Patients with risk scores of $\geq 5$ points were regarded as high risk. Patients who were treated according to the RAISE study protocol were defined as the RAISE group and the others were defined as the conventional group.

\section{Statistical analysis}

The Mann-Whitney $U$ test was performed to compare continuous variables between these two groups and Fisher's exact test for categorical variables. Differences were considered significant if $p<0.05$. Data are expressed as median and interquartile range. All calculations were performed with standard statistical software (JMP version 11 package for Windows, SAS, Cary, NC, USA).

\section{Results}

During the year of the study, 113 patients with KD were admitted to our hospital. After excluding five patients, one because their guardian refused consent and four for whom blood samples were not available, 108 patients were included in the study (Fig. 1).

Eleven patients $(10 \%)$ were in the positive group, and $97(90 \%)$ in the negative group. Of the eleven patients in the positive group, eight were positive only for antiYPT antibodies, one for only anti-YPM antibody and two for both (Table 1). Patients 7 (O:2b, 5b), 9 (O:5a, 5b), and $11(\mathrm{O}: 1 \mathrm{~b}, 2 \mathrm{~b})$ had increases in titers of more than two types of antibody.

Clinical manifestations of patients with KD according to serologically positive and negative groups are compared in Table 2 . The only significant differences between the two groups were in the incidence of CS (positive group, two patients [18 \%] vs negative group, 
Table 1 Characteristics of patients in positive group

\begin{tabular}{|c|c|c|c|c|c|c|c|}
\hline No & Age (months) & Sex & YPT & YPM & CS & Treatment group & Risk group \\
\hline 1 & 19 & Male & O6 (1:640) & Negative & $(-)$ & Conventional & Low \\
\hline 2 & 29 & Female & O6 (1:160) & Negative & $(-)$ & Conventional & Low \\
\hline 3 & 72 & Male & O3 (1:640) & Negative & $(-)$ & Conventional & Low \\
\hline 4 & 6 & Male & Negative & Positive & $(-)$ & Conventional & Low \\
\hline 5 & 66 & Male & O5b (1:160) & Negative & $(+)$ & Conventional & High \\
\hline 6 & 34 & Female & O6 (1:160) & Negative & $(-)$ & RAISE & Low \\
\hline 7 & 22 & Male & $\mathrm{O} 2 \mathrm{~b}, 5 \mathrm{~b}(1: 160)$ & Negative & $(+)$ & RAISE & High \\
\hline 8 & 70 & Male & O1a $(1: 160)$ & Negative & $(-)$ & RAISE & Low \\
\hline 9 & 21 & Female & O5a,5b (1:160) & Positive & $(-)$ & RAISE & Low \\
\hline 10 & 79 & Female & O6 (1:160) & Negative & $(-)$ & RAISE & High \\
\hline 11 & 21 & Female & $01 b, 2 b(1: 160)$ & Positive & $(-)$ & RAISE & Low \\
\hline
\end{tabular}

one patient [1\%]; $p=0.027$ ) and of abdominal symptoms (positive group, eight patients [73 \%] vs negative group, 33 patients [34 \%]; $p=0.020)$.

Although blood and/or stool cultures were performed in many patients in this study, YPT was not cultured from any of the samples.

Figures 2 and 3 show the medications and the number of patients overall, whose risk scores were $\geq 5$ points, in the conventional group and the RAISE group, respectively. Forty of 108 patients were treated according to the RAISE study protocol [15] (RAISE group). Ten patients in the RAISE group had positive risk scores and received IVIG + PSL therapy (Fig. 3). As shown in Table 3, there were no significant differences in clinical characteristics between patients in the RAISE and conventional groups. Two of 40 patients (5\%) in the RAISE group and one of $68(1.47 \%)$ in the conventional group had CS $(p=0.55)$. Further, two of 10 patients $(20 \%)$ with positive risk scores in the RAISE group (one YPT positive and one YPT negative) and one of 19 patients (5.26 \%) with positive risk scores in the conventional group (YPT positive) had CS $(p=0.27)$.

Table 2 Comparison of characteristics according to serologically positive and negative groups

\begin{tabular}{|c|c|c|c|}
\hline & Positive group $(n=11)$ & Negative group $(n=97)$ & $P$ value \\
\hline Sex (male/female) & $6 / 5$ & $61 / 36$ & 0.74 \\
\hline Age (months) & $29(21-70)$ & $24(13-49.5)$ & 0.26 \\
\hline Duration of fever (days) & $6(6-9)$ & $5(5-7)$ & 0.092 \\
\hline Frequency of administration of IVIG & $1(1-2)$ & $1(1-1)$ & 0.32 \\
\hline Acute-phase coronary artery dilation; $\mathrm{n}(\%)$ & $2(18.18 \%)$ & $4(4.12 \%)$ & 0.11 \\
\hline CS; n (\%) & $2(18.18 \%)$ & $1(1.03 \%)$ & 0.027 \\
\hline Abdominal symptoms; n (\%) & $8(72.73 \%)$ & $33(34.02 \%)$ & 0.02 \\
\hline White blood cell count $(/ \mu \mathrm{L})$ & $12,900(11,100-19,400)$ & $13,000(10,750-15,400)$ & 0.48 \\
\hline Absolute neutrophil count $(/ \mu \mathrm{L})$ & $8117(5741-24,654)$ & $8032(5696-10,240)$ & 0.73 \\
\hline Serum albumin concentration ( $\mathrm{g} / \mathrm{dL})$ & $3.8(3.4-4)$ & $3.7(3.4-4)$ & 0.54 \\
\hline Serum total bilirubin concentration (mg/dL) & $0.4(0.3-0.7)$ & $0.6(0.4-0.8)$ & 0.47 \\
\hline Serum aspartate aminotransferase (U/L) & $37(19-230)$ & $32.5(25-69.25)$ & 0.8 \\
\hline Serum sodium (mmol/L) & $134(133-138)$ & $136(134-138)$ & 0.24 \\
\hline Serum CRP concentration (mg/dL) & $4.36(1.81-8.09)$ & $7.09(3.09-11.72)$ & 0.27 \\
\hline Serum procalcitonin concentration (ng/mL) & $0.67(0.42-2.18)$ & $0.47(0.175-1.995)$ & 0.37 \\
\hline N-terminal pro-brain natriuretic peptide $(\mathrm{pg} / \mathrm{mL})$ & $379(81.75-771.5)$ & $386(140-936)$ & 0.74 \\
\hline Serum soluble interleukin-2 receptor (U/mL) & $1560(1350-2680)$ & $1635(1062.5-2157.5)$ & 0.37 \\
\hline Urinary $\beta$ 2-microglobulin/creatinine ratio & $101.9(10.7-203.7)$ & $18.9(5.9-126.3)$ & 0.25 \\
\hline High risk patients; n (\%) & $3(27.27 \%)$ & 26 (27.08 \%) & 1 \\
\hline
\end{tabular}

Values expressed as count (\%) for categorical variables and median (IQR) for continuous variables 


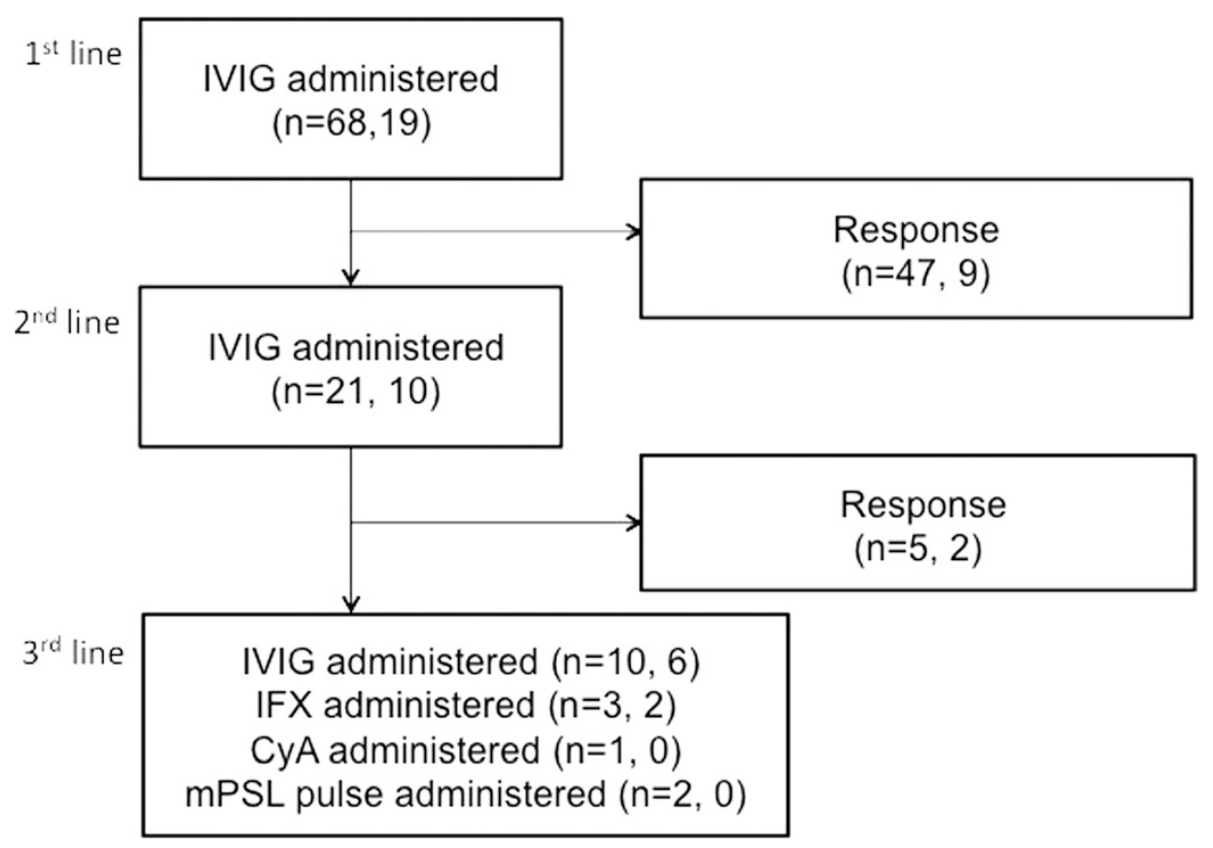

Fig. 2 Flow chart showing the distribution of study subjects and medications used for KD in the conventional group. The numbers in parentheses denote the overall number of patients in the conventional group, and the number of patients in the conventional group whose risk scores were $\geq 5$ points, respectively. IVIG: intravenous immunoglobulin, IFX: infliximab, CyA: cyclosporin, mPSL: methylprednisolone

These findings indicate that the RAISE study treatment protocol (IVIG + PSL for high-risk patients with no response to IVIG and IVIG for low-risk patients) did not decrease the frequency of CS in our cohort. Additionally, YPT infection did not affect risk scores of no response to IVIG in either RAISE or the conventional period.

\section{Discussion}

This is the first study to comprehensively investigate KD patients for evidence of YPT infection by assessing both anti-YPT antibodies for serotypes that can infect humans and YPM antibodies. We also analyzed the effect of the RAISE regimen to prevent CS development in KD.

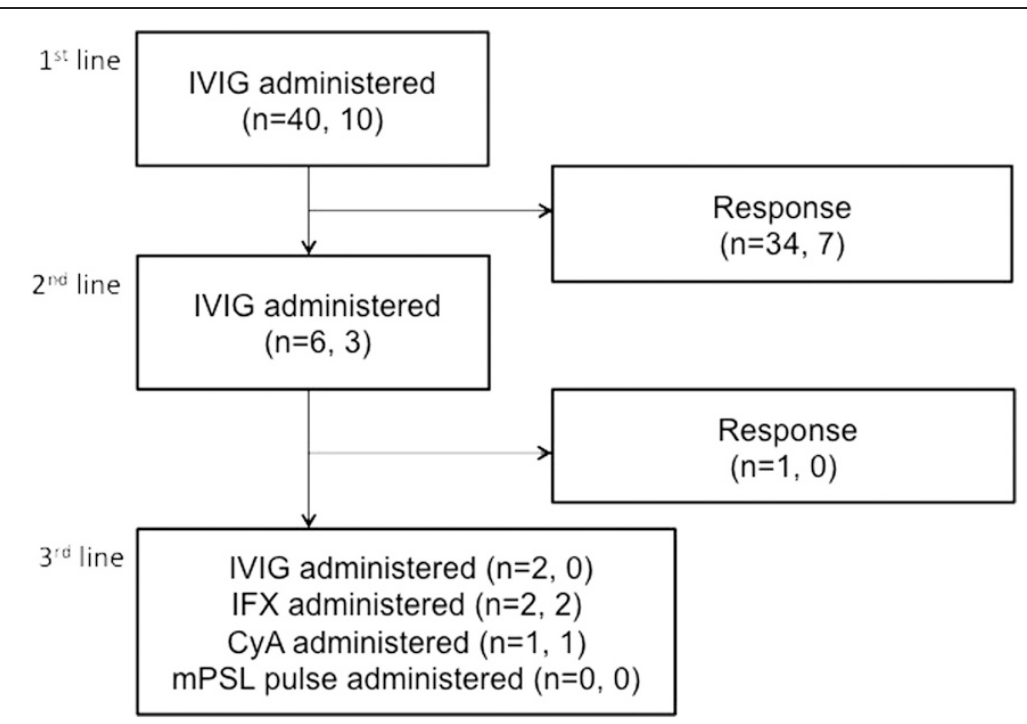

Fig. 3 Flow chart showing the distribution of study subjects and medications used for KD in the RAISE group. The numbers in parentheses denote the overall number of patients in the RAISE group, and the number in the RAISE group who received PSL because of risk scores $\geq 5$ points, respectively. IVIG: intravenous immunoglobulin, IFX: infliximab, CyA: cyclosporin, mPSL: methylprednisolone 
Table 3 Characteristics of patients according to study group

\begin{tabular}{|c|c|c|c|}
\hline & RAISE group $(n=40)$ & Conventional group $(n=68)$ & $P$ value \\
\hline Sex (male/female) & $23 / 17$ & $44 / 24$ & 0.54 \\
\hline Age (months) & $29(16.25-47.75)$ & $23(13-51.75)$ & 0.44 \\
\hline Duration of fever (days) & $5.5(5-6.75)$ & $6(5-8)$ & 0.21 \\
\hline Frequency of administration of IVIG & $1(1-1)$ & $1(1-2)$ & 0.11 \\
\hline Acute-phase coronary artery dilation; n (\%) & $3(7.5 \%)$ & $3(4.41 \%)$ & 0.67 \\
\hline CS; n (\%) & $2(5 \%)$ & $1(1.47 \%)$ & 0.55 \\
\hline Abdominal symptoms; $\mathrm{n}(\%)$ & $18(45 \%)$ & $23(33.82 \%)$ & 0.31 \\
\hline White blood cell count $(/ \mu \mathrm{L})$ & $13,250(11,700-17,200)$ & $12,700(10,200-15100)$ & 0.057 \\
\hline Absolute neutrophil count $(/ \mu \mathrm{L})$ & $8547(7195-11,520)$ & $7482(5356-10,086)$ & 0.057 \\
\hline Serum albumin concentration (g/dL) & $3.7(3.4-3.9)$ & $3.7(3.3-4)$ & 0.53 \\
\hline Serum total bilirubin concentration (mg/dL) & $0.6(0.4-0.775)$ & $0.6(0.4-0.8)$ & 1 \\
\hline Serum aspartate aminotransferase $(U / L)$ & $37(28-80.75)$ & $29(22-71)$ & 0.079 \\
\hline Serum sodium concentration (mmol/L) & $136(134-138)$ & $136(134-137)$ & 0.12 \\
\hline Serum CRP concentration (mg/dL) & $7.6(3.0925-12.545)$ & $6.36(2.52-11.08)$ & 0.41 \\
\hline Serum procalcitonin concentration $(\mathrm{ng} / \mathrm{mL})$ & $0.8(0.245-2.5125)$ & $0.405(0.1625-6.317)$ & 0.074 \\
\hline N-terminal pro-brain natriuretic peptide $(\mathrm{pg} / \mathrm{mL})$ & $336(146-811.5)$ & $510(130-983.5)$ & 0.55 \\
\hline Soluble interleukin-2 receptor $(\mathrm{U} / \mathrm{mL})$ & $1585(1087.5-2090)$ & $1660(1070-2360)$ & 0.59 \\
\hline Urinary $\beta$ 2-microglobulin/creatinine ratio & $19.36(5.92-151.9)$ & $21.57(5.97-130.74)$ & 0.98 \\
\hline YPT positive group & $6(15 \%)$ & $5(7.35 \%)$ & 0.32 \\
\hline High risk patients; n (\%) & $10(25 \%)$ & $19(27.9 \%)$ & 0.82 \\
\hline High risk YPT positive; n (\%) & $2(5 \%)$ & $1(1.47 \%)$ & 0.55 \\
\hline High risk patients with CS; n (\%) & $2(20 \%)$ & $1(5.26 \%)$ & 0.27 \\
\hline High risk, YPT positive, CS; n (\%) & $1(50 \%)$ & $1(100 \%)$ & 1 \\
\hline
\end{tabular}

Values expressed as count (\%) for categorical variables and median (IQR) for continuous variables

This study yielded four important clinical observations. First, 11/108 KD patients (10 \%) had positive titers of anti-YPT and/or anti-YPM antibodies. Second, the incidence of CS was significantly higher in the serologically positive (two patients, $18 \%$ ) than in the negative group (one patient, $1 \%)(p=0.027)$. Third, there were no clinical differences between the serologically positive and negative groups, except for the incidence of abdominal symptoms and CS. Fourth, in our cohort, the use of the RAISE study protocol, comprising IVIG + PSL therapy, did not decrease the frequency of CS in high-risk patients, neither did YPT infection influence sensitivity to treatment.

In 1983, Sato et al. reported that $2 / 12$ patients with YPT fulfilled the criteria for KD [20]. Since then, several other groups have confirmed an association between YPT infection and KD. Baba et al. reported that, from 1981 to $1989,29 / 329$ of their YPT patients fulfilled the criteria for KD [5]. In 2006, Tahara et al. reported that, from 1985 to 2004, 42/452 of their KD patients had bacteriological culture and/or serological evidence of YPT infection [7]. Although the percentage of KD patients with YPT infection in our study is similar to that in previous reports, the ratio may differ in other regions because geographical heterogeneity of YPT strains has been reported [21, 22].

We found increases in more than two types of antibodies in three patients (Patients 7, 9 and 11; Table 1). These findings may reflect-specific responses; however, two of these three patients were also positive for antiYPM antibodies (Patients 9 and 11), which makes nonspecific reactions unlikely. A search of published reports revealed no reports of cross-reacting antigens between different strains of YPT or of co-infection with more than two types of YPT in humans. Fukushima et al. described a patient infected with $\mathrm{O}$ serotype-untypeable strains with positive titers for two serological types of YPT [11]. Additionally, discrepancies between classical serotypes and O-genotypes have been reported [8]. Thus, there may be some new or untypeable strains of YPT.

Three of 11 patients in the serologically positive group (27\%) were positive for anti-YPM antibodies and two of three for both anti-YPT and anti-YPM antibodies. In 1993, Abe et al. demonstrated that YPT produce a superantigen, YPM [23, 24]. In 1995, Yoshino et al. reported that $95 \%$ of YPT strains in Japan harbor the ypm 
gene [21]. In 1997, Abe et al. reported detection of antiYPM antibodies in $61 \%$ of acute phase patients with YPT infection. Patients with systemic symptoms such as lymphadenopathy, transient renal dysfunction, and arthritis had significantly higher titers of anti-YPM than patients with gastrointestinal tract symptoms alone [14]. We speculate that checking for anti-YPM antibody would increase the rate of detection of YPT in KD patients. Further studies are required to ascertain whether this is true.

We found that the incidence of CS was significantly higher in the serologically positive group (two patients, $18 \%)$ than in the negative group (one patient, $1 \%)(p=$ 0.027). In 1997, Konishi et al. reported a patient with Kawasaki disease, coronary artery aneurysms, and documented YPT type $4 \mathrm{~b}$ infection confirmed by both microbiological and serological findings [4]. In 2006, Tahara et al. reported that $22 / 42 \mathrm{KD}$ patients with YPT infection had coronary artery lesions, this incidence being significantly higher than in the YPT-negative group [7]. In 2014, Kusuda et al. reported that serum KD-specific molecules were mostly derived from biofilms and possessed molecular structures common to microbe-associated molecular patterns from Bacillus cereus, Bacillus subtilis, YPT and Staphylococcus aureus. They also reported that extracts from Bacillus cereus, Bacillus subtilis, YPT and Staphylococcus aureus induced proinflammatory cytokines by human coronary artery endothelial cells [25]. This pathogenesis can contribute to the higher incidence of CS in cases of KD associated with YPT.

As shown in recent published studies, new medications such as infliximab, cyclosporin, and methylprednisolone have been developed [18, 26-29]. These have reduced the incidence of CS in KD patients; however, there is still a $2.8 \%$ incidence of CS in Japan ([http:// www.jichi.ac.jp/dph/kawasakibyou/20130909/mcls22report. pdf] (in Japanese)). Our findings suggest that YPT infection is a major risk factor for CS in KD patients even after the adoption of these newly developed treatment strategies.

In this study, the only clinical distinction between KD patients with and without YPT infection was in the incidence of abdominal symptoms and CS. Of note, YPT is usually very sensitive to most antibiotics $[6,30]$. Antibiotics for Group-A streptococcal infection reduce the incidence of acute rheumatic fever [31]. In the future, we should aim at conducting research to clarify if antibiotic treatment for YPT infection could prevent the development of KD or reduce the incidence of CS after rapid diagnostic techniques for YPT are developed, such as the loop-mediated isothermal amplification method [32].

In our cohort, treatment with IVIG + PSL, as described in the RAISE study, did not decrease the frequency of CS in high-risk patients. In 2012, Kobayashi et al. reported the findings of a randomized, open-label, blinded-endpoints trial in which 248 high-risk patients participated (the RAISE study). They found a significantly lower incidence of CS in patients who received IVIG + PSL therapy than in those who received conventional IVIG therapy [15]. After the publication of this study [15], the use of steroids has been spreading for the standard treatment of $\mathrm{KD}$ and the use of steroids are recommended for the cases who did not respond to initial treatment with IVIG as written in KD treatment guideline in Japan [18]. The recent national survey in Japan revealed that among patients not responding to IVIG therapy, $29.0 \%$ of them were treated with steroids [19]. However, it is not a routine practice in other countries because the evidence for the use of steroids is generally lacking in non-Japanese populations [33, 34]. Although RAISE protocol is widely prevalent in Japan, further larger randomised controlled trials are required to evaluate the use of corticosteroid therapy for both adjunctive primary treatment in high-risk cases and for additional 'rescue' therapy in IVIG non-responsive cases [33].

In our cohort, there were fewer high-risk patients $(n=29)$ and only ten of them received IVIG + PSL. However, two of the ten high-risk patients who received IVIG + PSL therapy had CS, indicating that even this treatment strategy did not completely prevent CS in all high-risk KD patients.

One limitation of our study is that no YPT was cultured from patients' blood and/or stool samples after they had been kept in cold enrichment cultures at $27{ }^{\circ} \mathrm{C}$. This may be attributable to antibiotic administration before obtaining the samples, which occurred in about $60 \%$ of our KD patients. Thus, our study lacks definitive evidence of YPT infection in the patients with KD who were serologically positive. Another limitation is that, although this was a single-center study, our therapeutic strategy changed during its course because of the publication of the RAISE study, which showed clear evidence of the efficacy of steroids for KD. Further, this study included relatively few YPT positive patients overall and relatively few high-risk patients.

\section{Conclusions}

We documented that $10 \%$ of our KD patients had positive anti-YPT and/or anti-YPM antibody titers and $18 \%$ of serologically positive patients had CS, meaning that YPT infection is associated with a very high-risk of CS in patients with KD. We failed to detect any other clinical differences between serologically positive and negative patients, aside from gastrointestinal symptoms. We also reported that treatment with IVIG + PSL as reported by the RAISE study did not decrease the frequency of CS in this study. However, our sample size 
was overly small to draw such conclusions. Further investigation in a larger cohort is necessary to confirm our findings.

Though many studies on KD treatment have been performed, few of them have focused on the cause of this condition. Further studies are needed to clarify the effect of YPT infection on KD and identify the cause of KD and its optimal treatment. Furthermore, detection of YPT infection and specific treatment in the early phase could reduce the risk of CS.

\section{Abbreviations}

KD: Kawasaki disease; YPT: Yersinia pseudotuberculosis; YPM: Y. pseudotuberculosisderived mitogen; CS: Cardiac sequelae; RAISE: Randomized controlled trial to Assess Immunoglobulin plus Steroid Efficacy for Kawasaki disease; VIG: IntraVenous ImmunoGlobulin; CRP: C-reactive protein; PSL: Prednisolone.

\section{Competing interests}

Kazumoto lijima has received grants from Pfizer Japan, Takeda Pharmaceutical, Asahi Kasei Pharma, Japan Blood Product Organization, Novartis Pharma K.K., and Mitsubishi Tanabe Pharma and lecture fees from Novartis Pharma K.K., Asahi Kasei Pharma, and Pfizer Japan.

\section{Authors' contributions}

$\mathrm{TH}$ and $\mathrm{KI}$ designed the study concept and wrote the manuscript. KN interpreted the data and wrote the manuscript. $\mathrm{KH}, \mathrm{YI}$ and $\mathrm{MK}$ collected and interpreted the data. JA and HN critically reviewed the manuscript. All authors read and approved the final version of the manuscript.

\section{Acknowledgement}

We appreciate all of our patients and their gurdians who gave us the consent to participate in this study.

This study was supported in part by a Grant for Child Health and Development (22-A1) from the Ministry of Health, Labour and Welfare to JA and Grant from Okayama Prf. Research Center of Environment and Public Health to HN.

\section{Author details}

'Department of Pediatrics, Japanese Red Cross Society Himeji Hospital, Hyogo, Japan. ${ }^{2}$ Department of Pediatrics, Kobe University Hospital, Kobe, Japan. ${ }^{3}$ Department of Allergy and Immunology, National Research Institute for Child Health and Development, Tokyo, Japan. ${ }^{4}$ Department of Bacteriology, Okayama Prefectural Institute for Environmental Science and Public Health, Okayama, Japan.

Received: 10 November 2014 Accepted: 23 October 2015 Published online: 11 November 2015

\section{References}

1. Saundankar J, Yim D, Itotoh B, Payne R, Maslin K, Jape G, et al. The epidemiology and clinical features of Kawasaki disease in Australia. Pediatrics. 2014;133:e1009-14

2. Huang SK, Lin MT, Chen HC, Huang SC, Wu MH. Epidemiology of Kawasaki disease: prevalence from national database and future trends projection by system dynamics modeling. J Pediatr. 2013;163:126-31. e121.

3. Uehara R, Belay ED. Epidemiology of Kawasaki disease in Asia, Europe, and the United States. J Epidemiol. 2012;22:79-85.

4. Konishi N, Baba K, Abe J, Maruko T, Waki K, Takeda N, et al. A case of Kawasaki disease with coronary artery aneurysms documenting Yersinia pseudotuberculosis infection. Acta Paediatr. 1997;86:661-4.

5. Baba K, Takeda N, Tanaka M. Cases of Yersinia pseudotuberculosis infection having diagnostic criteria of Kawasaki disease. Contrib Microbiol Immunol. 1991;12:292-6

6. Sato K, Ouchi K, Komazawa M. Ampicillin vs. placebo for Yersinia pseudotuberculosis infection in children. Pediatr Infect Dis J. 1988:7:686-9.

7. Tahara M, Baba K, Waki K, Arakaki Y. Analysis of Kawasaki disease showing elevated antibody titres of Yersinia pseudotuberculosis. Acta Paediatr. 2006;95:1661-4.
8. Bogdanovich T, Carniel E, Fukushima H, Skurnik M. Use of O-antigen gene cluster-specific PCRs for the identification and O-genotyping of Yersinia pseudotuberculosis and Yersinia pestis. J Clin Microbiol. 2003;41:5103-12.

9. Bogdanovich TM, Carniel E, Fukushima H, Skurnik M. Genetic (sero) typing of Yersinia pseudotuberculosis. Adv Exp Med Biol. 2003;529:337-40.

10. Hayashidani HT I. Yersinia pseudotuberculosis infection. Modern media (in Japanese). Modern Media. 2005;51:211-6.

11. Carniel E. The Yersinia high-pathogenicity island. Int Microbiol. 1999:2:161-7.

12. Carnoy C, Mullet C, Muller-Alouf H, Leteurtre E, Simonet M. Superantigen YPMa exacerbates the virulence of Yersinia pseudotuberculosis in mice. Infect Immun. 2000;68:2553-9.

13. Cornelis GR, Biot T, Lambert de Rouvroit C, Michiels T, Mulder B, Sluiters C, et al. The Yersinia yop regulon. Mol Microbiol. 1989;3:1455-9.

14. Abe J, Onimaru M, Matsumoto S, Noma S, Baba K, Ito Y, et al. Clinical role for a superantigen in Yersinia pseudotuberculosis infection. J Clin Invest. 1997;99:1823-30

15. Kobayashi T, Saji T, Otani T, Takeuchi K, Nakamura T, Arakawa H, et al. Efficacy of immunoglobulin plus prednisolone for prevention of coronary artery abnormalities in severe Kawasaki disease (RAISE study): a randomised, open-label, blinded-endpoints trial. Lancet. 2012;379:1613-20.

16. Ayusawa M, Sonobe T, Uemura S, Ogawa S, Nakamura Y, Kiyosawa N, et al. Revision of diagnostic guidelines for Kawasaki disease (the 5 th revised edition). Pediatr Int. 2005:47:232-4.

17. Paff JR, Triplett DA, Saari TN. Clinical and laboratory aspects of Yersinia pseudotuberculosis infections, with a report of two cases. Am J Clin Pathol. 1976;66:101-10

18. Research Committee of the Japanese Society of Pediatric C, Cardiac Surgery Committee for Development of Guidelines for Medical Treatment of Acute Kawasaki D. Guidelines for medical treatment of acute Kawasaki disease: report of the Research Committee of the Japanese Society of Pediatric Cardiology and Cardiac Surgery (2012 revised version). Pediatr Int. 2014;56:135-58

19. Group JCSJW. Guidelines for diagnosis and management of cardiovascular sequelae in Kawasaki disease (JCS 2013). Digest version. Circ J. 2014;78:2521-62.

20. Sato K, Ouchi K, Taki M. Yersinia pseudotuberculosis infection in children, resembling Izumi fever and Kawasaki syndrome. Pediatr Infect Dis. 1983:2:123-6.

21. Fukushima H, Matsuda $Y$, Seki R, Tsubokura M, Takeda N, Shubin FN, et al. Geographical heterogeneity between Far Eastern and Western countries in prevalence of the virulence plasmid, the superantigen Yersinia pseudotuberculosis-derived mitogen, and the high-pathogenicity island among Yersinia pseudotuberculosis strains. J Clin Microbiol. 2001;39:3541-7.

22. Yoshino K, Ramamurthy T, Nair GB, Fukushima H, Ohtomo Y, Takeda N, et al. Geographical heterogeneity between Far East and Europe in prevalence of ypm gene encoding the novel superantigen among Yersinia pseudotuberculosis strains. J Clin Microbiol. 1995:33:3356-8.

23. Abe J, Takeda T, Watanabe Y, Nakao H, Kobayashi N, Leung DY, et al. Evidence for superantigen production by Yersinia pseudotuberculosis. J Immunol. 1993;151:4183-8.

24. Uchiyama T, Miyoshi-Akiyama T, Kato H, Fujimaki W, Imanishi K, Yan XJ. Superantigenic properties of a novel mitogenic substance produced by Yersinia pseudotuberculosis isolated from patients manifesting acute and systemic symptoms. J Immunol. 1993;151:4407-13.

25. Kusuda T, Nakashima Y, Murata K, Kanno S, Nishio H, Saito M, et al. Kawasaki disease-specific molecules in the sera are linked to microbe-associated molecular patterns in the biofilms. PLoS One. 2014;9, e113054.

26. Burns JC, Mason WH, Hauger SB, Janai H, Bastian JF, Wohrley JD, et al. Infliximab treatment for refractory Kawasaki syndrome. J Pediatr. 2005;146:662-7.

27. Ogata S, Ogihara Y, Honda T, Kon S, Akiyama K, Ishii M. Corticosteroid pulse combination therapy for refractory Kawasaki disease: a randomized trial. Pediatrics. 2012;129:e17-23.

28. Tremoulet AH, Pancoast $P$, Franco A, Bujold M, Shimizu C, Onouchi $Y$, et al. Calcineurin inhibitor treatment of intravenous immunoglobulin-resistant Kawasaki disease. J Pediatr. 2012;161:506-12. e501.

29. Wright DA, Newburger JW, Baker A, Sundel RP. Treatment of immune globulin-resistant Kawasaki disease with pulsed doses of corticosteroids. J Pediatr. 1996;128:146-9.

30. Lemaitre BC, Mazigh DA, Scavizzi MR. Failure of beta-lactam antibiotics and marked efficacy of fluoroquinolones in treatment of murine Yersinia 
pseudotuberculosis infection. Antimicrob Agents Chemother. 1991;35:1785-90.

31. Robertson KA, Volmink JA, Mayosi BM. Antibiotics for the primary prevention of acute rheumatic fever: a meta-analysis. BMC Cardiovasc Disord. 2005;5:11.

32. Horisaka T, Fujita K, Iwata T, Nakadai A, Okatani AT, Horikita T, et al. Sensitive and specific detection of Yersinia pseudotuberculosis by loop-mediated isothermal amplification. J Clin Microbiol. 2004:42:5349-52.

33. Etoom Y, Banihani R, Finkelstein Y. Critical review of: Efficacy of immunoglobulin plus prednisone for prevention of coronary artery prednisolone for prevention of coronary abnormalities in severe Kawasaki disease (RAISE study): a randomized, open-label, blinded-endpoints trial. J Popul Ther Clin Pharmacol. 2013:20:e91-4.

34. Yim D, Curtis N, Cheung M, Burgner D. An update on Kawasaki disease II: clinical features, diagnosis, treatment and outcomes. J Paediatr Child Health. 2013:49:614-23.

\section{Submit your next manuscript to BioMed Central and take full advantage of:}

- Convenient online submission

- Thorough peer review

- No space constraints or color figure charges

- Immediate publication on acceptance

- Inclusion in PubMed, CAS, Scopus and Google Scholar

- Research which is freely available for redistribution 